\title{
BOAS-TYPE INEQUALITY FOR 3-CONVEX FUNCTIONS AT A POINT
}

\author{
Josip PeČArić, Dora PoKaz AND MARJAn PRALJaK
}

Abstract. Starting from a very general form of Boas-type inequality from [5] we get Boas-type inequality for 3 -convex functions at a point. For special $\lambda$-balanced sets, weight functions and measures we derive various examples.

Mathematics subject classification (2010): Primary 26D10; Secondary 26D15.

Keywords and phrases: Boas inequality, 3-convex function at a point.

\section{REFERENCES}

[1] I. A. BAloch, J. PeČARIĆ, M. Praljak, Generalization of Levinson's inequality, J. Math. Inequal. 9 (2) (2015), 571-586

[2] R. P. BoAs, Some integral inequalities related to Hardy's inequality, J. Anal. Math. 23 (1970), 53-63.

[3] A. ČIŽmeŠIJa, S. Huss Ain, AND J. PeČARIĆ, Some new refinements of Hardy and Pólya-Knopp's inequalities, J. Funct. Spaces Appl. 7 (2) (2009), 167-186.

[4] A. ČIŽMeŠIJA, J. PeČArić, AND L.-E. Persson, On strengthened Hardy and Pólya-Knopp's inequalities, J. Approx. Theory 125 (2003), 74-84.

[5] A. ČIŽMEŠIJA, J. PEČARIĆ, D. POKAZ, A new general Boas-type inequality and related Cauchy-type means, Math. Inequal. Appl., 12 (3) (2012), 599-617.

[6] S. Kaijser, L. Nikolova, L. E. Persson, And A. Wedestig, Hardy-Type Inequalities via Convexity, Math. Inequal. Appl., 8 (2005), 403-417.

[7] N. Levinson, Generalizations of an Inequality of Hardy, Duke Math. J. 31 (1964), 389-394.

[8] D. LuOR, Modular inequalities for the Hardy-Littlewood averages, Math. Inequal. Appl. 13 (3) (2010), 635-642.

[9] K. Krulić Himmelreich, J. PeČarić, D. Pokaz, M. Praljak, New results about Hardy-type inequality, J. Math. Inequal., 9 (4) (2015), 1259-1269.

[10] J. PeČARIĆ, M. PraljaK, A. WitKows Ki, Generalized Levinson's inequality and exponential convexity, Opuscula Math., 35 (1) (2015), 397-410.

[11] J. PeČarić, Frank Proschan, Y. L. Tong, Convex Functions, Partial Orderings and Statistical Applications, Academic Press, San Diego, 1992. 